\title{
Chapter 22 \\ SBM Guide to the Literature as of June 1972
}

\author{
Rolf Hagedorn
}

\begin{abstract}
A large number of research papers motivated Rolf Hagedorn to prepare a guide to the Statistical Bootstrap Model literature in mid-1972; he wanted to show the reader a) which publications best prepare the uninitiated, b) how different publications relate to each other, and c) he aimed to connect similar developments into groups. The two figures classify in this fashion the research works, providing in the rendition the relevant Refs. [[1]-[70]]. The editor updated all preprint citations for the 2015 printing.
\end{abstract}

This guide contains two figures and a list of references. It is neither complete nor unbiased.

Figure 22.1 gives the recommended reading sequence. After reading the introductory lectures (partly overlapping), the reader should be able to enter the lower boxes at any place, though it might be advantageous to follow the given sequence. The reader will notice overlaps and inconsistencies, because the reading sequence does not coincide with the historical development.

Figure 22.2 tries to picture the logical (and roughly the historical) connections. The isolated box with the name Koppe indicates that he was the first to contemplate statistical and thermodynamical interpretations of pion production. Unfortunately, his two papers came too early and went unnoticed. When two years later Fermi elaborated the same idea in great detail, he obviously had no knowledge of Koppe's work.

In both figures, the sequence goes along top to bottom lines of connection, unless indicated otherwise by an arrow.

Preprint CERN-TH 1535, dated 20 July 1972, see: http://cds.cern.ch/record/961894?ln=en not intended as formal publication.

R. Hagedorn: (deceased) CERN-TH, 1211 Geneve 23, Switzerland

J. Rafelski ( $₫)$

Department of Physics, The University of Arizona, Tucson, AZ 85721, USA

(C) The Author(s) 2016

J. Rafelski (ed.), Melting Hadrons, Boiling Quarks - From Hagedorn Temperature

to Ultra-Relativistic Heavy-Ion Collisions at CERN,

DOI 10.1007/978-3-319-17545-4_22 


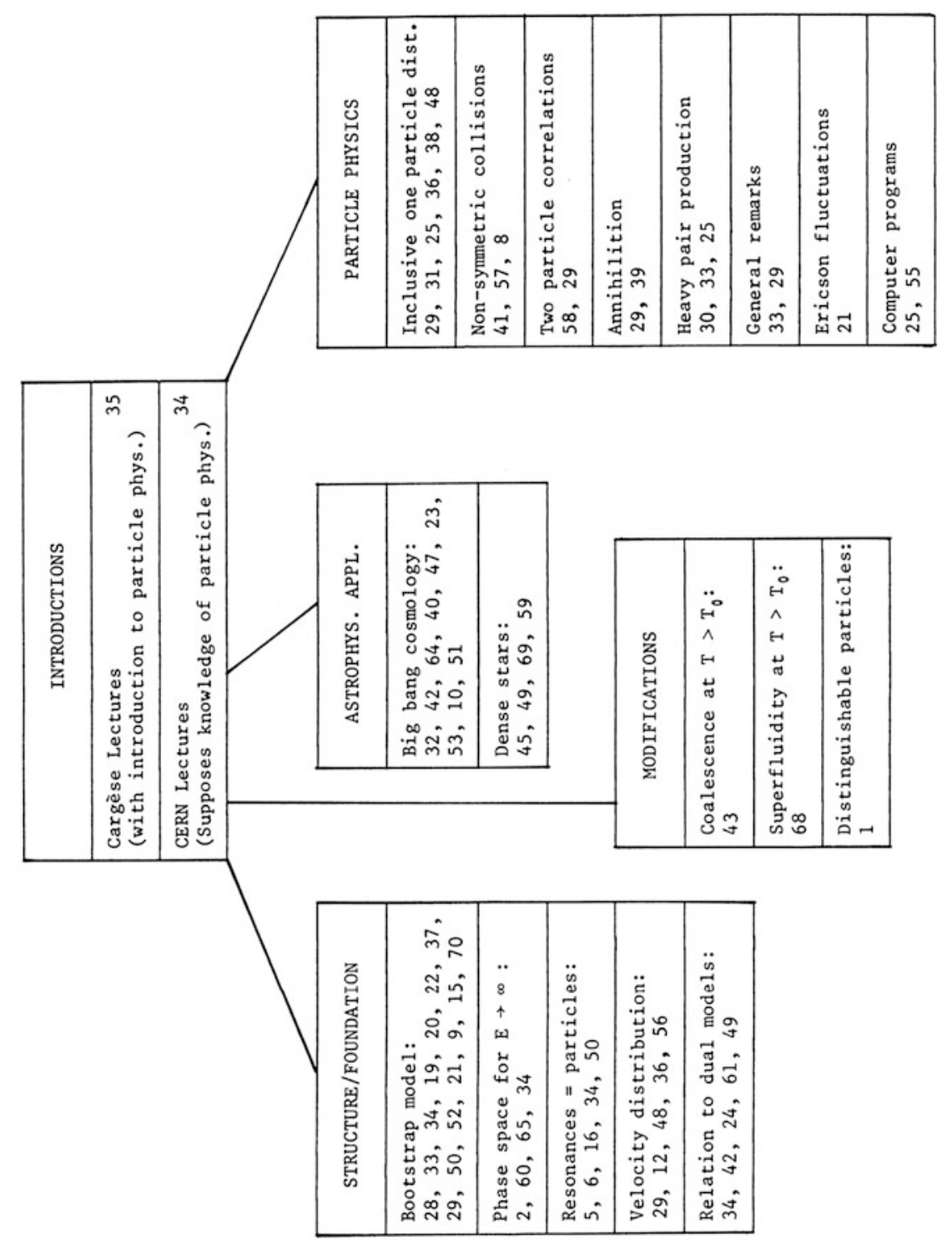

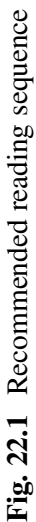




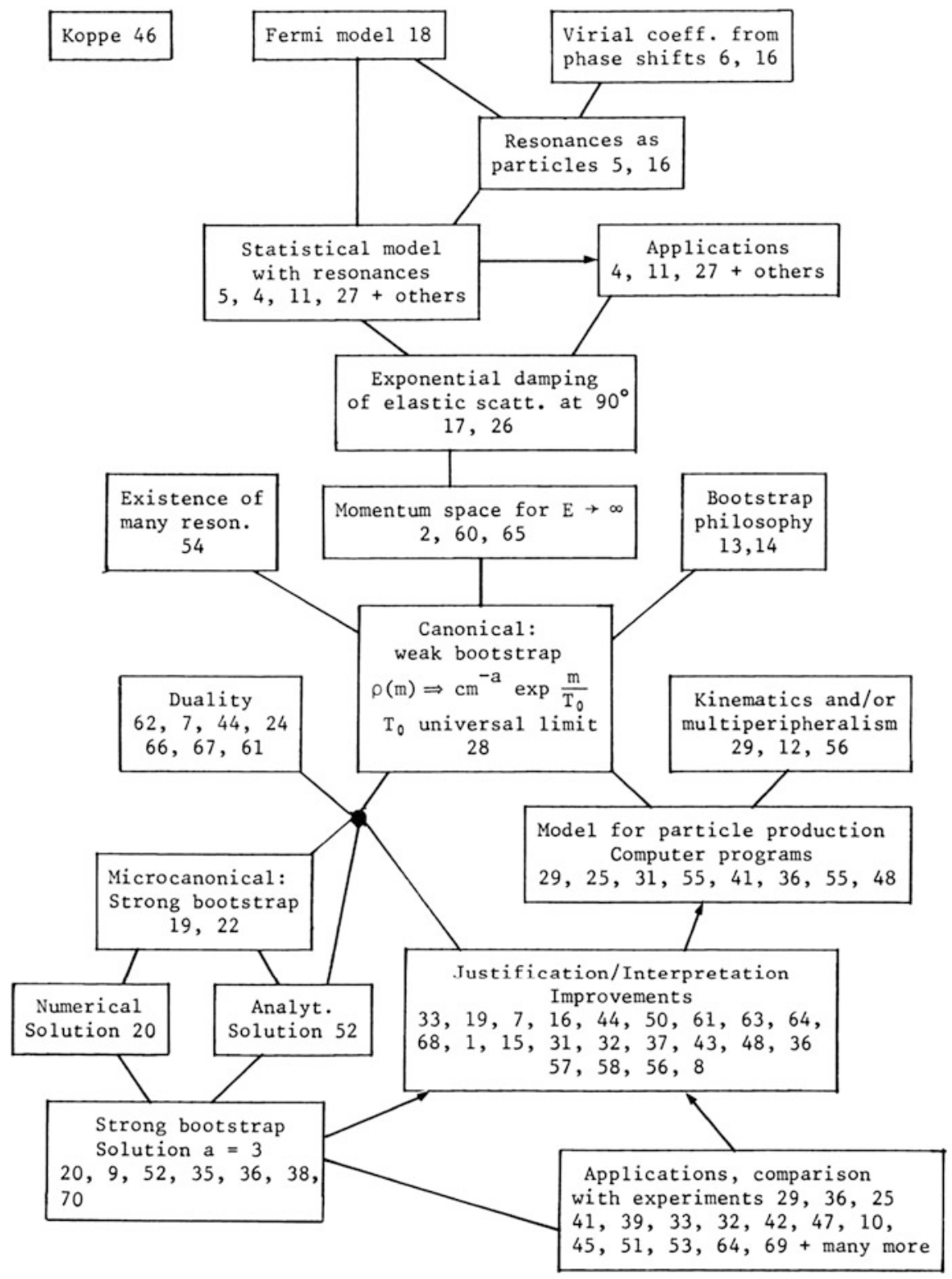

Fig. 22.2 Logical connections with reference numbers 
Open Access This book is distributed under the terms of the Creative Commons Attribution Noncommercial License which permits any noncommercial use, distribution, and reproduction in any medium, provided the original author(s) and sources are credited.

\section{References}

1. M. Alexanian, Phys. Rev. D 5, 922 (1972)

2. G. Auberson, B. Escoubès, Nuovo Cimento 36, 628 (1965)

3. J.N. Bahcall, S.C. Frautschi, Astrophys. J. 170, L81 (1971)

4. V.S. Barashenkov, B.M. Barbashev, E.G. Bubelov, V.M. Maksimenko, Nucl. Phys. 5, 17 (1958)

5. S.Z. Belenkij, Nucl. Phys. 2, 259 (1956)

6. E. Beth, G.E. Uhlenbeck, Physica 4, 915 (1937)

7. R. Brout, Letter to R. Hagedorn, March 1969 (unpublished)

8. B. Buschbeck, J. Hödl, Nucl. Phys. B 48, 288 (1972)

9. R.D. Carlitz, Phys. Rev. D 5, 3231 (1972)

10. R.D. Carlitz, C.S. Frautschi, W. Nahm, Astron. Astrophys. 26, 171 (1973)

11. F. Cerulus, Nuovo Cimento 14, 827 (1959)

12. F. Cerulus, Proceedings of the Colloquium on High Multiplicity Hadron Interactions, Ecole Polytechnique, Paris, 1970

13. G.F. Chew, S. Mandelstam, Nuovo Cimento 19, 752 (1961)

14. G.F. Chew, Phys. Today 23, 23 (1970)

15. C.B. Chiu, R.L. Heimann, Phys. Rev. D 4, 3184 (1971)

16. R. Dashen, S. Ma, H.J. Bernstein, Phys. Rev. 187, 349 (1959)

17. G. Fast, R. Hagedorn, Nuovo Cimento 27, 208 (1963); G. Fast, R. Hagedorn, L.W. Jones, Nuovo Cimento 27, 856 (1963)

18. E. Fermi, Prog. Theor. Phys. 5, 570 (1950)

19. S.C. Frautschi, Phys. Rev. D 3, 2821 (1971)

20. S.C. Frautschi, C.J. Hamer, Phys. Rev. D 4, 2125 (1971)

21. S.C. Frautschi, Nuovo Cimento A 12, 133 (1972)

22. S.C. Frautschi, Lectures Given at the Torini Colloquium on Thermodynamics of Strong Interactions; forthcoming report of the Institute of Theoretical Physics, University of Torino (1972)

23. S.C. Frautschi, G. Steigmann, J. Bahcall, Astrophys. J. 175, 307 (1972)

24. S. Fubini, D. Gordon, G. Veneziano, Phys. Lett. B 29, 679 (1969); S. Fubini, G. Veneziano, Nuovo Cimento A 64, 811 (1969)

25. H. Grote, R. Hagedorn, J. Ranft, Atlas of Particle Spectra (CERN, 1970)

26. R. Hagedorn, Nuovo Cimento 35, 216 (1965)

27. R. Hagedorn, Nuovo Cimento 15, 434 (1960)

28. R. Hagedorn, Suppl. Nuovo Cimento 3, 147 (1965)

29. R. Hagedorn, J. Ranft, Suppl. Nuovo Cimento 6, 169 (1968)

30. R. Hagedorn, Suppl. Nuovo Cimento 6, 311 (1968)

31. R. Hagedorn, Nuovo Cimento A 56, 1027 (1968)

32. R. Hagedorn, Astron. Astrophys. 5, 184 (1970)

33. R. Hagedorn, Nucl. Phys. B 24, 93 (1970)

34. R. Hagedorn, Thermodynamics of Strong Interactions. Yellow Report CERN-71-12 (1971)

35. R. Hagedorn, Lectures to be Published in the Proceedings of the Cargèse Summer School on Physics of the Early Universe and Cosmology, Cargèse, 1971

36. R. Hagedorn, J. Ranft, Nucl. Phys. B 48, 157 (1972)

37. R. Hagedorn, Lectures given at the Torino Colloquium on Thermodynamics of Strong Interactions; forthcoming report of the Institute of Theoretical Physics, University of Torino (1972) 
38. R. Hagedorn, Lecture to be published in The Proceedings of the Third International Colloquium on Many-Body Reactions, Zakopane, 1972

39. C.J. Hamer, Nuovo Cimento A 12, 162 (1972)

40. E.R. Harrison, Phys. Rev. D 1, 2726 (1970)

41. H. Than, J. Ranft, Lett. Nuovo Cimento 5, 655 (1972)

42. K. Huang, S. Weinberg, Phys. Rev. Lett. 25, 895 (1970)

43. K. Imaeda, Nuovo Cimento Lett. 1, 290 (1971)

44. A. Krzywicki, Phys. Rev. 187, 1964 (1969)

45. K. Koebke, E. Hilf, R. Ebert, Nature 226, 625 (1970)

46. H. Koppe, Z. Naturforsch. A 3, 251 (1968); H. Koppe, Phys. Rev. 76, 688 (1949)

47. W. Kundt, Springer Tracts Mod. Phys. 58, 1 (1971)

48. J. Letessier, A. Tounsi, Nuovo Cimento A 11, 353 (1972)

49. Y.C. Leung, G.G. Wang, Astrophys. 170, 499 (1971); H. Lee, Y.C. Leung, C.G. Wang, Astrophys. J. 166, 387 (1971)

50. G. Matthiae, Nucl. Phys. B 7, 142 (1948)

51. C. Mollenhoff, Astron. Astrophys. 7, 488 (1970)

52. W. Nahm, Nucl. Phys. B 45, 525 (1972)

53. R. Omnès, Phys. Rep. C 3, 1 (1972)

54. Particle Data Group, Phys. Lett. B 33, 1 (1970)

55. J. Ranft, Leipzig University Report TUL 37 (1970), reprinted as 'Reprint 5' in [25]

56. G. Ranft, J. Ranft, Phys. Lett. B 32, 207 (1970)

57. J. Ranft, E. Matthäus, Phys. Lett. B 40230 (1972)

58. G. Ranft, J. Ranft, Phys. Lett. B 40, 131 (1972); G. Ranft, J. Ranft, Inclusive two-particle correlations from the thermodynamic model. Preprint CERN-TH-1532

59. C.E. Rhoades, R. Ruffini, Astrophys. Lett. 163, L83 (1971)

60. H. Satz, Nuovo Cimento 37, 1407 (1965)

61. H. Satz, Lecture to be Published in Proceedings of the Colloquium on High Multiplicity Hadron Interactions, Zakopane, 1972

62. C. Schmid, Phys. Rev. Lett. 20, 628 (1968)

63. L. Sertorio, M. Toller, Nuovo Cimento A 14, 21 (1973)

64. D. Stauffer, Phys. Rev. D 6, 1797 (1972)

65. J. Vandermeulen, Bull. Soc. R. Sci. Liège (Belgium) 34e $(1 / 2), 34$ (1965)

66. G. Veneziano, Nuovo Cimento A 57, 190 (1968)

67. G. Veneziano, Phys. Today 22, 31 (1969)

68. A. Mann, R. Weiner, Nuovo Cimento Lett. 2, 248 (1971); A. Mann, R. Weiner, Phys. Lett. B 40, 383 (1972)

69. J.C. Wheeler, Astrophys. J. 169, 105 (1971)

70. J. Yellin, Nucl. Phys. B 52, 583 (1973) 


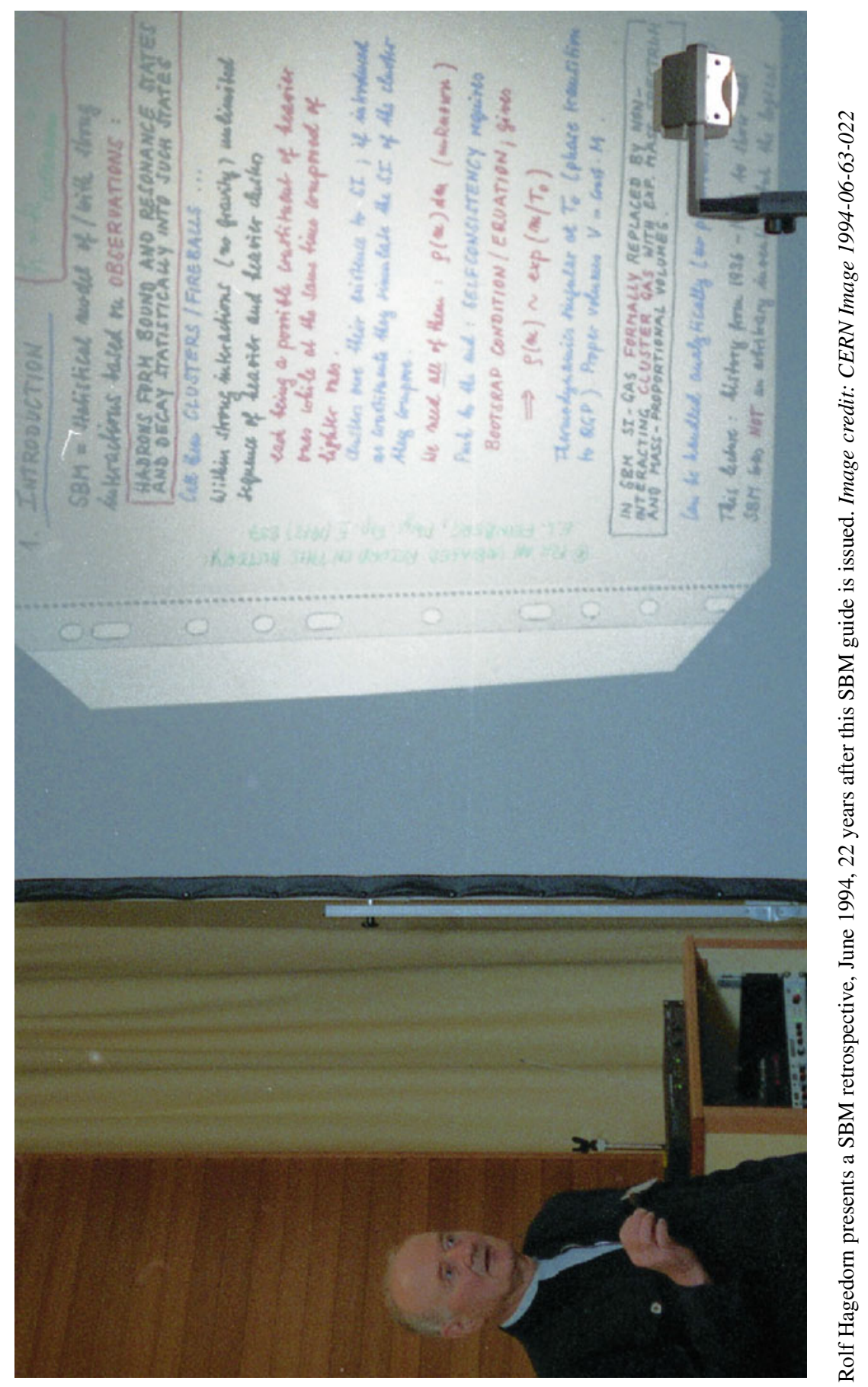

